\title{
Ageísmo e COVID-19: revisão integrativa
}

\author{
Ageism and COVID-19: an integrative review \\ Edadismo y COVID-19: revisión integradora
}

\section{Resumo}

Esta pesquisa traz revisão integrativa sobre o tema "Ageísmo durante a pandemia de COVID-19", buscando responder à questão se existiu eclosão do ageísmo durante a pandemia ou apenas se tornaram visíveis estereótipos já presentes na sociedade. A revisão seguiu os passos dispostos no protocolo PRISMA- Principais Itens para Relatar Revisões Sistemáticas e Meta-análises, tendo selecionado 34 artigos entre os meses de agosto a outubro de 2020 e abril a junho de 2021. Nota-se recrudescimento de estereótipos já presentes contra o grupo etário composto por pessoas idosas, o qual foi tratado de forma homogênea. As pessoas idosas foram retratadas como um fardo para a sociedade e amplamente associadas, principalmente por governos, profissionais de saúde, mídias e redes sociais ao declínio, inutilidade e dependência, atitudes estas que reforçaram embates intergeracionais, preconceitos e discriminação. Entretanto, manifestações de empatia também surgiram, em contraposição a estas posições. Destaca-se que, em um mundo onde as vulnerabilidades sociais, o acesso aos cuidados e a determinação social do adoecer se tornaram especialmente visíveis pela pandemia, é importante enfrentar firmemente o ageísmo em todas as suas formas, oferecendo esperança para com os grupos em desvantagem social. Destaca-se, por último, o pouco interesse que o tema despertou para a literatura acadêmica nacional, sendo muito mais amplamente analisado e discutido fora do Brasil, como o observado nesta revisão.

Palavras-chave: Ageísmo; Idosos; COVID-19.

\begin{abstract}
This research brings an integrative review on the theme "Ageism during the COVID-19 pandemic", seeking to answer the question whether there was an outbreak of ageism during the pandemic or simply the visibility of stereotypes already presents in society. The review followed the steps provided by the PRISMA protocol - Key Items for Reporting Systematic Reviews and Meta-analyzes having selected 34 articles from August to October 2020 and from April to June 2021. The resurgence of stereotypes already presents against the age group composed of elderly people is observed, which was treated homogeneously. Portrayed as a burden to society, the elderly was widely associated with decline, uselessness, and dependence mainly by governments, health professionals, the media, and social networks. Such attitudes reinforced intergenerational conflicts, prejudice, and discrimination. However, manifestations of empathy also emerged in contrast to those positions. It is noteworthy that, in a world where social vulnerabilities, access to care and the social determination of falling ill have become especially visible by the pandemic, it is important to firmly confront ageism in all its forms, offering hope for groups in social disadvantage. Finally, we highlight the little interest that the topic aroused in the national academic literature, being much more widely analyzed, and discussed outside Brazil, as observed in this review.
\end{abstract}

Keywords: Ageism; Elderly; COVID-19.

\section{Resumen}

Esta investigación trae una revisión integradora sobre el tema "edadismo durante la pandemia COVID-19", buscando responder a la pregunta de si hubo un brote de discriminación por edad durante la pandemia o solo se hicieron visibles 
los estereotipos ya presentes en la sociedad. La revisión siguió los pasos dispuestos en el protocolo PRISMA Elementos Clave para Informar Revisiones Sistemáticas y Metaanálisis y se seleccionaron 34 artículos entre los meses de agosto a octubre de 2020 y abril a junio de 2021. Se observa un recrudecimiento de estereotipos ya presentes contra el grupo de edad compuesto por personas mayores, lo cual fue tratado de manera homogénea. Las personas mayores fueron retratadas como una carga para la sociedad y ampliamente asociadas, principalmente por gobiernos, profesionales de la salud, medios de comunicación y redes sociales, con el declive, la inutilidad y la dependencia, actitudes que reforzaron los enfrentamientos intergeneracionales, los prejuicios y la discriminación. Sin embargo, también surgieron manifestaciones de empatía, en contraste con estas posiciones. Es de destacar que, en un mundo donde las vulnerabilidades sociales, el acceso a la atención y la determinación social de enfermarse se han vuelto especialmente visibles por la pandemia, es importante enfrentar con firmeza el edadismo en todas sus formas, ofreciendo esperanza a los grupos en desventaja social. Finalmente, destacamos el escaso interés que despertó el tema en la literatura académica nacional, siendo mucho más analizado y discutido fuera de Brasil, como se observa en esta revisión.

Palabras clave: Edadismo; Personas mayores; COVID-19.

\section{Introdução}

Com a emergência da pandemia de COVID-19, causada pelo vírus SARS-COV-2, o mundo vem experimentado, além da infecção causada pelo microrganismo, um incremento nas manifestações de vários tipos de estereótipos, preconceitos e discriminações.

Estereótipos são interpretações construídas na cultura que permitem uma análise através de crenças pré-formadas. Por serem pré-formadas, estas crenças moldam a visão e definição de mundo antes mesmo que este possa ser observado, reproduzindo-se e mantendo-se no senso comum mediante códigos, tradições e posições sociais. Compartilhados coletivamente, promovem categorizações e estigmatizações a partir de atributos, características ou traços -psicológicos, morais ou físicos- conferidos a um agrupamento humano, por meio da aplicação de um ou mais critérios, como por exemplo, idade, sexo, orientação sexual, filiação religiosa, dentre outros. Desta forma, influenciam condutas e comportamentos em interações sociais (Moscovici, 2009).

Nem sempre negativos, estereótipos sociais são generalizações que permitem distinguir grupos sociais: os que pertencem a um dado grupo e os que a este não pertencem. Quando associados às emoções e juízos de valor, originam estruturas psíquicas mais complexas, traduzindo-se em atitudes e preconceitos. É a combinação de estereótipos positivos ou negativos aos sentimentos, de aceitação ou rejeição, que levam à discriminação. Esta última, pode ser praticada por apenas uma pessoa, ou tornar-se uma norma social implícita ou prática institucionalizada. Estas representações adquirem vida própria nas coletividades, reproduzindo-se, extinguindo-se ou dando origem a novas, porém influenciando comportamentos, tanto individuais quanto coletivos (Teixeira, Souza, \& Maia, 2018; Moscovici, 2009).

A espécie humana se viu, em vários momentos de sua história, frente a pandemias. Em todas estas fases de adoecimento e morte, que afetaram milhões de pessoas, os estereótipos, o preconceito e a discriminação contra determinados grupos humanos recrudesceram. Desta forma, judeus, afrodescendentes, orientais, ciganos, homossexuais, dentre outros grupos, foram sendo culpabilizados, simultaneamente ou não, como causadores destas afecções, o que resultou em marginalização, exclusão, agressões físicas e até genocídio, com base na visão de que estes grupos, com características diversas do grupo hegemônico, seriam os responsáveis pelo quadro epidemiológico. Acreditava-se, entretanto, que, com a modernidade, a crença na ciência pouparia a espécie humana destas manifestações de intolerância, mas não é o que se tem observado. Se nota que, em tempos de recursos escassos, como o que caracteriza a atual pandemia, ameaças simbólicas e disputas entre valores e crenças se exacerbam (Ayalon, 2020; Guérios, 2020).

A presença de estereótipos negativos relacionados à idade é denominada ageísmo, considerada uma das formas mais socialmente normalizadas de discriminação, principalmente quando voltada contra a pessoa idosa. É termo inicialmente desenvolvido pelo gerontólogo americano Robert Butler, em 1969, e que apresenta aspectos inter-relacionados em três 
dimensões (estereótipos, preconceito e discriminação), três manifestações (institucional, interpessoal e autodirigido) e duas formas de expressão (explícita e implícita). Além disso, o ageísmo interage com outros preconceitos, tais como: sexismo (principalmente quanto à aparência física), o racismo estrutural e o capacitismo (ao assumir que a deficiência é sinônimo de envelhecimento) (Organização Mundial de Saúde [OMS], 2021).

$\mathrm{O}$ aspecto institucional do ageísmo contra a pessoa idosa se refere às leis, políticas e regras que prejudicam o indivíduo; o interpessoal consiste no desrespeito social e, por fim, o autodirigido, que trata da internalização do preconceito. É fenômeno que ocorre em todas as esferas da convivência social, como no trabalho (desde o recrutamento até o processo de demissão e aposentadoria), na habitação (no momento da escolha dos inquilinos, ao selecionarem os mais jovens que buscam residências em contratos mais curtos), na tecnologia (ao deduzir que idosos são incapazes de aprender este novo meio de comunicação). Também está presente na área da saúde, na educação e no setor financeiro, obstaculizando o acesso a serviços (Cox, 2020). Mas, é na mídia, ao retratar os idosos de maneira homogênea, ignorando suas particularidades, que este mais se manifesta (Manso, Mello, \& Lopes, 2018).

O ageísmo já havia sido exacerbado em outras emergências sanitárias anteriores à COVID-19, como documentado quando da ocorrência da onda de calor de 1995 em Chicago, onde o capital social limitado (dado pela quantidade e qualidade de laços formais e informais- comunidade, amigos, família) se mostrou como um potencializador de estereótipos contra a pessoa idosa (Ayalon, 2020). Em estudo realizado por Donizzetti (2019), com a aplicação de um questionário a 886 moradores da Itália, a fim de verificar a percepção destas pessoas sobre o processo de envelhecimento e a presença de estereótipos negativos em relação à pessoa idosa, os resultados mostraram tanto desconhecimento como ansiedade com o envelhecer, principalmente entre as mulheres pesquisadas, sendo estes fatores associados à presença de ageísmo.

Muito se tem falado sobre a 'eclosão' de estereótipos negativos contra a pessoa idosa na época da pandemia de COVID-19, mas será que efetivamente houve esta "eclosão" ou o preconceito etário naturalizado, presente nas diversas sociedades, foi claramente exposto? Diante destes fatos, propôs-se esta revisão, com o objetivo de responder a esta pergunta.

\section{Metodologia}

Trata-se de revisão integrativa da literatura, realizada durante os meses de agosto a outubro de 2020 e abril a junho de 2021, nas bibliotecas virtuais BVS (Biblioteca Virtual em Saúde) e PubMed (US National Library of Medicine's), nas bases de dados LILACS (Literatura Latino-americana e do Caribe em Ciências da Saúde) e MedLine (Medical Literature Analysis and Retrieval System Online).

Os Descritores em Ciência e Saúde (DeCS) selecionados foram idosos, idosos 80 e mais, ageísmo, idadismo, preconceito etário; e na língua inglesa, os Medical Subject Headings (MeSH): aged, ageism. Estes descritores foram associados com o operador booleano "AND" e com o descritor COVID-19.

Após a busca, foram encontrados 71 artigos no total, nas linguas portuguesa, inglesa e espanhola, os quais foram submetidos a um processo de verificação e análise, conforme disposto no protocolo PRISMA- Principais Itens para Relatar Revisões Sistemáticas e Meta-análises (Moher, Liberati, Tetzlaff, Altman [PRISMA Group], 2009), como se observa na Figura 1. Os artigos passaram por um processo inicial de verificação e triagem, primeiramente através da leitura e a análise dos títulos e resumos, excluindo-se artigos que não atenderam à proposta deste estudo. Posteriormente, foi realizado o cruzamento entre as bases para retirar os duplicados, restando apenas 34 artigos. Estes passos foram realizados em programa Word- Microsoft Office 365®. A seguir os artigos selecionados foram novamente confrontados com os critérios de inclusão e exclusão e avaliados por todos os autores desta revisão, não havendo discordâncias quanto à sua inclusão. Destaca-se a quantidade de artigos encontrados em pré-print, justificados pela necessidade de divulgação rápida do conhecimento a respeito da pandemia, traduzindo tendência cientifica mundial. 
Figura 1 - Fluxograma dos artigos encontrados e selecionados por meio da pesquisa na base de dados.

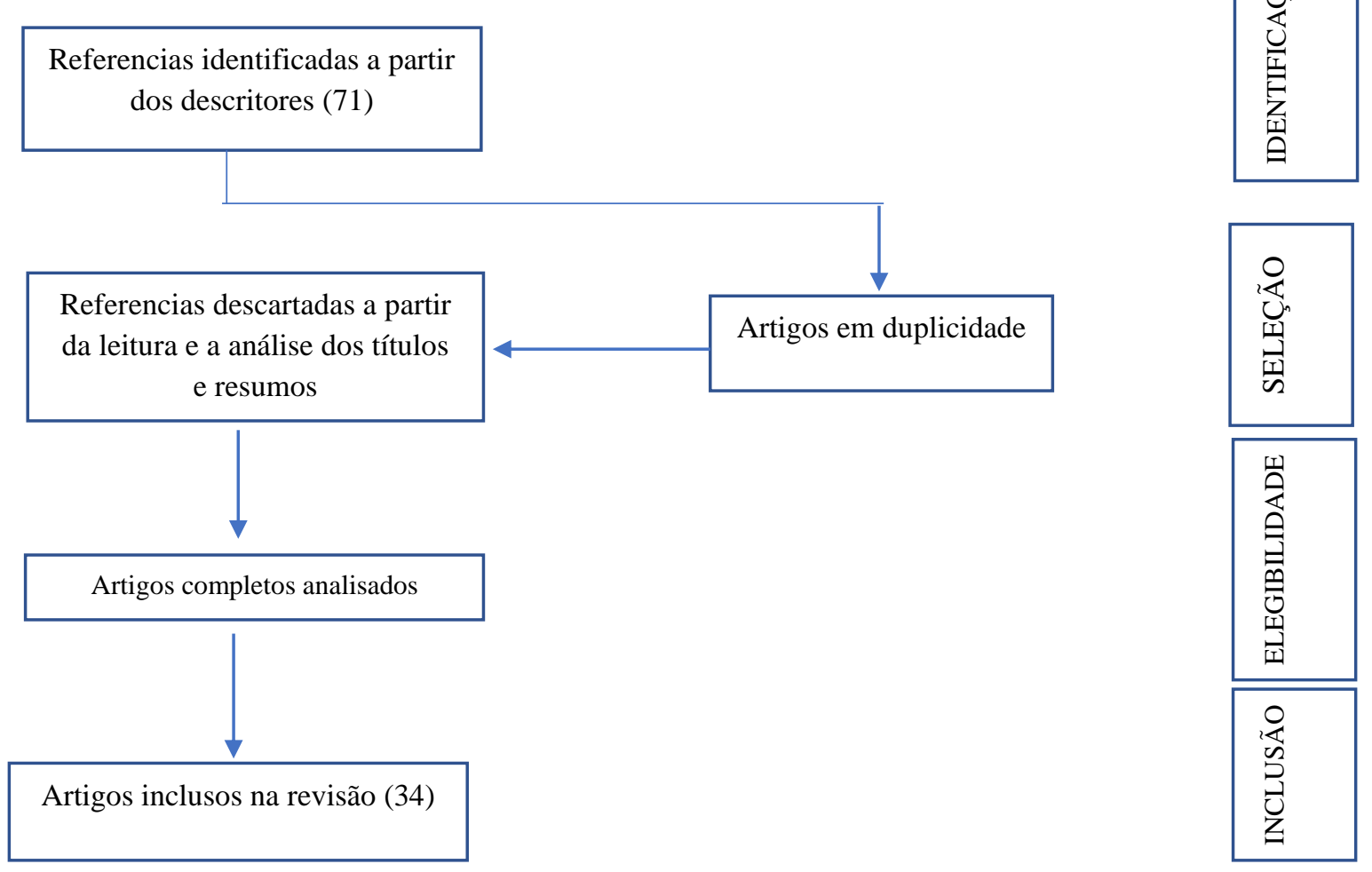

Fonte: Autores.

\section{Resultados e Discussão}

Não foram apenas os mais velhos as únicas vítimas da pandemia de COVID-19, mas a ênfase e a forma como aconteceu o debate sobre a mortalidade causada pela doença efetivamente exacerbaram e tornaram claras atitudes negativas para com a pessoa idosa com sérias consequências, atuais e futuras, que ainda deverão ser avaliadas, como enfatizam os 34 artigos elegíveis para esta revisão.

O ageísmo não se trata de fenômeno novo, muito menos surpreendente, como comentado, e se encontra ligado à visão de que a velhice é necessariamente uma fase de declínios cognitivos acentuados e doenças físicas. Desta forma, o segmento etário idoso passa a ser tratado como um grupo homogêneo, aplicando-se atributos de fragilidade e dependência a toda pessoa idosa.

Estes atributos são empregados frequentemente como justificativa para a exclusão das pessoas e traz consigo a mensagem que o envelhecer é apenas declínio, devendo estas pessoas idosas dar, necessariamente, lugar aos mais jovens. Estes argumentos foram os mais comumente aplicados na pandemia de COVID-19, fundamentando ações governamentais, diretrizes médicas e reações sociais. Estes estereótipos levaram ao aprofundamento de tensões intergeracionais (Ayalon, 2020; Colenda et al., 2020).

Um dos aspectos que a literatura destaca para um possível incremento do ageísmo, ou apenas como desencadeante de sua explicitação, relaciona-se à própria vulnerabilidade das pessoas idosas à pandemia, com maior necessidade de recursos de saúde incluindo mais dias de internação e maior demanda de cuidados intensivos e apoio ventilatório.

Isto foi traduzido em um argumento frequentemente utilizado que apresenta a pessoa idosa gerando um custo social desnecessário, já que são pessoas que apresentam baixa produtividade e menor potencial para contribuir economicamente para a sociedade. Desta forma, foram cobradas dos governos medidas que priorizassem adultos jovens e aqueles de meia-idade em 
detrimento aos idosos na assistência à pandemia. Estas observações foram reproduzidas por líderes políticos de alguns países e pela mídia, sempre com boa aceitação social (Academia Francesa de Medicina, 2020; Bravo-Segal \& Villar, 2020; D’Cruz \& Banerjee, 2020; Reynolds, 2020).

Este ageísmo político eclodiu em locais como Austrália, E.U.A e no Reino Unido, como demonstra Lichtenstein (2020), o qual ressalta que o ageísmo tomou formas semelhantes nos três países, apesar destes terem medidas diferentes de enfrentamento à pandemia. Analisando notícias da mídia destes três países, durante os meses de abril a junho de 2020, o autor identificou como frequentes afirmações de que os idosos irritam muito seus parentes, xingamentos contra pessoas idosas e defesa de segregação. $\mathrm{O}$ isolamento indefinido para os idosos foi amplamente aceito por estas sociedades, com discurso de que toda uma coorte de pessoas havia vivido além da sua utilidade para a sociedade e enriquecido às custas das gerações futuras.

Diretrizes governamentais foram implantadas em vários países, tais como China, Israel e Reino Unido, obrigando a contenção das pessoas idosas no domicílio, independentemente de seu estado de saúde, de suas necessidades ou da presença de rede colaborativa (Academia Francesa de Medicina, 2020) No Brasil, a determinação de distanciamento físico acabou por penalizar aqueles mais necessitados, com menor rede de apoio e em situação de vulnerabilidade (Hammerschmidt, Bonatelli, \& Carvalho, 2020; Silva et al., 2020). Idosos que se deslocaram para compras de mantimentos ou para buscar cuidados médicos foram vítimas de crimes de ódio em alguns locais, já que violavam as diretrizes governamentais de isolamento (D'Cruz \& Banerjee, 2020).

Vários países emitiram comunicados semelhantes ao lançado pelo governo inglês no início da pandemia, solicitando que os maiores de 70 anos se auto isolassem em casa, enquanto os demais grupos etários deveriam seguir vida normal. $\mathrm{O}$ argumento utilizado no comunicado governamental destacava que as pessoas idosas já têm suas pensões e, portanto, não sentiriam nenhum impacto financeiro com o isolamento, tendo a obrigação de se retirarem da sociedade dando lugar aos mais jovens. Em Israel, um ex-ministro da saúde comentou que não se pode permitir que "muitas poucas pessoas, cuja vida útil não é alta, arruínem o país", sendo que o Estado deve sacrificá-las. Mensagens como estas foram ouvidas em vários outros países (Ayalon, 2020)

Ayaloan et al. (2020) destacam que estes discursos governamentais coincidem com uma visão de que toda pessoa acima de 70 anos é igualmente frágil e incapaz de contribuir para a sociedade. Imagem reforçada pela mídia e redes sociais, desprezando dados científicos que demonstram a heterogeneidade do grupo idoso, que difere em suas origens culturais, genéticas e histórias de vida e saúde. A ciência já demonstrou que o envelhecer é altamente diversificado e dependente do contexto e que incontáveis idosos realizam contribuições importantes para a sociedade.

A atual pandemia de COVID-19 trouxe à tona um discurso político que desvaloriza os mais velhos, vistos como um custo social, questionando o valor de suas vidas e desconsiderando sua contribuição para a sociedade. Curiosamente, uma boa parte dos políticos que trouxeram este discurso, são eles próprios idosos, o que parece demonstrar o quanto a velhice ainda não é desejada e até negada. Discursos de políticos que se consideram atletas, que se mantem jovens apesar da idade, foram veiculados e reproduzidos pela mídia e pelas redes sociais de forma acrítica, penalizando e culpabilizando principalmente as pessoas mais velhas que, durante sua trajetória, foram expostos a condições de vida e trabalho que os levaram a ter um envelhecimento com mais desvantagens e vulnerabilidades biopsicossociais. Lembrando que foram estes idosos mais pobres, em sua maioria afrodescendentes, com maior número de comorbidades, os mais afetados pela pandemia (Cox, 2020).

Todas estas notícias e falas reproduzem a visão de que os idosos devem se retirar da sociedade para dar lugar aos mais jovens, traduzindo assim a disputa intergeracional sobre recursos materiais em situações de escassez (Ayaloan, 2020) Autores destacam que não houve apenas distanciamento físico para o idosos, mas sim um verdadeiro distanciamento social, principalmente em sociedades já extremamente divididas do ponto de vista geracional como são as sociedades europeias. Estas 
divisões já vinham se acentuando antes mesmo da pandemia, desencadeadas por movimentos como o Brexit por exemplo, onde as pessoas idosas foram frequentemente acusadas por "roubar" o futuro das pessoas mais novas (Fraser et al., 2020).

$\mathrm{Na}$ China, a tensão entre gerações se exacerbou quando inúmeros idosos se recusaram a utilizar máscaras faciais, sendo dado destaque ao egoísmo inerente a todas as pessoas idosas. Mas, quando idosos pobres morreram e doaram seus parcos recursos para o combate à doença, o segmento etário idoso foi descrito como altruísta, salientando-se que é um grupo capaz de morrer pelos demais (Xiang et al., 2020)

Os grandes potencializadores na propagação de estereótipos, ageísmo e atitudes negativas para com as pessoas mais velhas, acentuando assim a exclusão e o preconceito nesta pandemia, foram a mídia e as redes sociais. Um artigo documental exploratório peruano analisou como a mídia jornalística do país retratou o idoso nas primeiras semanas de isolamento durante a pandemia. Observou-se que os estereótipos mais comuns foram que os idosos são doentes, tem deficiências físicas e cognitiva, possuem dependência econômica, são sozinhos e lhes falta autonomia e independência, tratando todos as pessoas com amis de 60 anos desta forma (Ângulo-Giraldo et al., 2020).

Expressões tais como a hashtag \#BoomerRemover foram amplamente reproduzidas nas redes sociais, destacando que, em momentos como os da pandemia é socialmente aceitável pedir aos idosos para morrer (Brooke \& Jackson, 2020; D'Cruz \& Banerjee, 2020; Fraser et al., 2020; Lichtenstein, 2020; Monahan, MacDonald, Lytle, Apriceno, Levy, 2020; Wister \& Speechley, 2020). Esta expressão veiculada nas redes sociais e reproduzida de outras formas em vários países, incluindo o Brasil, trazia a afirmação de que as pessoas idosas são irresponsáveis, teimosas e pouco solidárias (Fraser et al., 2020; Tarazona-Santalbina, Martinez-Velilla, Vidán, Garcia-Navarro, 2020).

Retratar a pandemia como um problema apenas dos idosos foi um dos aspectos que surgiram no início da pandemia e que se perpetuaram. Reproduzindo um discurso de que os idosos são alvos preferenciais da COVID-19 e que as altas taxas de mortes entre este segmento populacional eram inevitáveis e normais, destacavam que jovens saudáveis eram imunes, estimulando assim a realização de festas e aglomerações, conhecidas como "Festas Corona", as quais ocorreram em inúmeros países, independentemente das medidas restritivas adotadas pelos governos (Ayaloan et al., 2020; Fraser et al., 2020; Wister \& Speechley, 2020; Xiang et al., 2020).

Este discurso de 'nós' (jovens) versus 'eles' (os idosos) predominou em vários momentos, acentuando a divisão etária, colocando gerações umas contra as outras e favorecendo o incremento de reações de ageísmo (Ayaloan et al., 2020; D’Cruz \& Banerjee, 2020). Em vários momentos, as redes sociais e as mídias associaram a COVID-19 a uma doença de idosos, o que distorceu os fatos, piorando, inclusive, a propagação do vírus (Fraser et al., 2020; Wister \& Speechley, 2020).

Analisando 82.893 dados do Twiter ${ }^{\circledR}$ entre 23 de janeiro e 20 de maio de 2020 versando sobre idosos e COVID 19, pesquisadores encontraram conteúdos ageístas sob a categoria de opiniões pessoais, mas também foram veiculadas piadas; ridicularizações; memes depreciativos e com conteúdo desvalorizante; afirmações de que a vida dos idosos valia menos, e de que a pandemia tinha o lado bom de diminuir o número de idosos (Xiang et al., 2020).

Várias opiniões nas redes sociais apoiaram o isolamento do idoso, sendo que um estudo demonstrou que estas mensagens se associaram, em sua maioria, a mensagens que minimizavam a pandemia (Xiang et al., 2020). Resultados semelhantes foram encontrados por outro grupo de pesquisadores após analisar 18.128 tweets entre os dias 12 e 21 de março (Jimenez-Sotomayor; Gomez-Moreno, \& Soto-Perez-de-Celis, 2020)

Idosos foram encorajados pelas redes sociais a renunciarem ao uso de ventiladores, a fim de que estes pudessem ser utilizados pelos mais jovens. Quando isto ocorria, os fatos eram divulgados amplamente, sempre destacando o quanto estes idosos já haviam vivido e que deveriam ser exemplos para as demais pessoas idosas (Barret, Michael, \& Padavic, 2020)

Não só nas redes sociais se visualizou este fenômeno. Em pesquisa feita em dois jornais espanhóis durante a fase mais crítica da pandemia no país, analisando 501 manchetes relacionadas aos idosos, se notou que este grupo foi representado 
desfavoravelmente, como sendo homogêneo, extremamente vulnerável, sendo a pessoa idosa associada ao declínio e dependência, o que justificaria práticas discriminatórias (Bravo-Segal \& Villar, 2020). Em outra pesquisa feita com notícias veiculadas pelas mídias americanas, canadenses, italianas, espanholas, alemãs, finlandesas, israelenses, além de outras vindas da Índia e de Cingapura, observou-se que todas mostraram os idosos como indefesos, frágeis e incapazes de contribuir para a sociedade (Ayalon et al., 2020). Estudo brasileiro com notícias veiculadas em jornal de grande circulação, destaca a ênfase dada por estes aos estereótipos negativos (Leão, Ferreira \& Faustino, 2020).

A literatura acadêmica destaca ainda a forma com a mídia noticiou a morte de idosos: quando jovens ou adultos jovens morriam, longas reportagens destacavam estes fatos, enquanto as mortes de idosos somente eram apresentadas em números frios e totalizados (Academia Médica Francesa, 2020; Fraser et al., 2020; Tarazona-Santalbina, Martinez-Velilla, \& Vidán, 2020; Wister \& Speechley, 2020). Este tratamento foi responsável por normalizar as mortes de pessoas idosas, reforçando, desta forma, o ageísmo. Pouco destaque foi dado pela imprensa a centenários que se recuperaram da COVID-19 (Ayalon, 2020).

Nas redes sociais, outro tipo de movimento ageísta também foi observado, desta vez pautado no denominado ageísmo compassivo ou benevolente, resultante da fusão entre percepções positivas e românticas sobre o envelhecimento combinadas com atributos outorgados aos idosos tais como incompetência, fragilidade, dependência, passividade, vitimização, em um misto de estereótipos. Isto resultou em ações paternalistas caracterizadas por comportamentos de ajuda nem sempre desejados pelos idosos ou até necessários (Vervaecke \& Meisner, 2020).

O movimento "carmongering" é citado como exemplo deste ageísmo compassivo. Este movimento se espalhou pelas redes sociais, com um script de apoio à dependência, sem considerar que nem todo idoso necessita de auxílio. Muitos destes grupos de ajuda foram divulgados pelo aplicativo TikTok®, sendo interpretados como uma "ajuda performática", onde indivíduos socialmente privilegiados registraram e compartilharam ações de auxílio que nem sempre tinham consentimento dos idosos, reforçando o estereótipo de fragilidade de um grupo que não pode se cuidar ou gerir sua vida sozinho. Autores destacam as relações de poder e autoafirmação midiática envolvidas nestas comunidades virtuais ditas altruístas, voltadas não apenas para idosos, mas também para outros grupos vulneráveis (Vervaecke \& Meisner, 2020)

As redes sociais, entretanto, não foram utilizadas apenas para veicular discursos ageistas, mas também foram de extrema valia para amenizar o distanciamento físico, evitando-se o afastamento social. Redes como Zoom®, Facebook®, Whatsapp ${ }^{\circledR}$, entre outras puderam permitir ao idosos ficarem conectados com sua família e amigos (Ayalon, 2020). No Reino Unido, vários aplicativos como o Nextdoor App® ${ }^{\circledR}$, uma plataforma para vizinhos e comunidades, incentivou conversas e conexões entre pessoas e terapias on line foram disponibilizados para os grupos que necessitaram de distanciamento (Brooke \& Jackson, 2020). Idosos em exclusão digital relataram mais solidão, sendo que a maioria destas pessoas idosas são também os mais socialmente desfavorecidos, não só com restrições de acesso aos meios digitais, mas também com outras restrições de acesso (Cox, 2020; D'Cruz \& Banerjee, 2020)

Estudo realizado com tweets ( $\mathrm{n}=188)$ em 23 de março de 2020, nos E.U.A, demonstrou que, apesar do ageísmo estar solidamente enraizado na cultura popular americana, pessoas se opuseram às afirmativas "boomer removal", afirmando que as vidas dos idosos tem valor, em um movimento de solidariedade intergeracional (Barret, Michael, \& Padavic, 2020).

Houve ainda uma série de medidas de empatia aos idosos restritos às suas casas, como a abertura do comercio em horários especiais somente para atendê-los e sinais inequívocos de solidariedade intergeracional, com jovens providenciando e se voluntariando para compra de mantimentos e medicamentos, auxiliando as pessoas idosas a se manterem conectadas com seus entes queridos através de smartfones, tanto em casa como nos hospitais (Fraser et al., 2020; Wister \& Speechley, 2020). O fortalecimento da solidariedade entre gerações reduz o ageísmo, daí a importância de fomentar estas trocas. Deve-se ressaltar 
que pesquisas recentes destacam que os jovens, principalmente aqueles com atitudes negativas quanto ao envelhecer, estavam mais propensos que os idosos a relatar maior angústia, solidão e sofrimento psicológico durante a pandemia (Ayalon, 2020).

Várias sociedades médicas demonstraram e ressaltaram como os idosos, principalmente médicos aposentados e outros profissionais de saúde, se engajaram no atendimento à população e como suas habilidades, desenvolvidas ao longo da vida, foram de extrema relevância para a atenção aos infectados graves. Os idosos também apresentaram mais práticas positivas de saúde mesmo quando em situações de distanciamento (Academia Francesa de Medicina, 2020; Colenda et al., 2020; Previtali, Allen, \& Varlamova, 2020)

O impacto do ageísmo sobre a saúde da pessoa idosa é considerável. Quando as pessoas idosas incorporam estereótipos negativos relacionados ao envelhecer, estes trazem impacto, principalmente, sobre as respostas emocionais ao estresse (Ayalon, 2020). Pesquisa realizada em Israel com 888 idosos, durante a pandemia de COVID-19, mostrou que o ageísmo impactou na vida dos idosos do país, causando maior ansiedade e percepção de ser um fardo para a sociedade (CohnSchwartz \& Ayalon, 2020). Outra pesquisa demonstrou a correlação entre a presença de ageísmo e sintomas de ansiedade em idosos, verificando que que quanto maior o nível do primeiro, maior a segunda (Bergman, Cohen-Fridel, Shrira, Bodner, Palgi, 2020). Com o distanciamento social, houve incremento do número de quadros depressivos e de ansiedade, má qualidade do sono e na redução de atividade física e de estímulos cognitivos para os idosos (Sepúlveda-Loyola et al., 2020).

$\mathrm{O}$ ageísmo traz um senso de inutilidade às pessoas mais velhas, afetando seriamente sua autoestima e bem-estar, o que as torna vulneráveis ao isolamento social e a desfechos adversos em saúde, com aumento não só do risco de ansiedade e depressão, mas também de disfunção cognitiva, doenças cardíacas e maior mortalidade (Ayalon, 2020; Brooke \& Jackson, 2020). Também é responsável por doenças neurológicas ou afecções de saúde mental, dentre outras, mas, o que mais preocupa, é o ageísmo dos profissionais de saúde e o autoageísmo, que normaliza determinadas doenças e sintomas, as quais passam a ser atribuídas ao próprio processo de envelhecimento, atuando como barreiras ao acesso à serviços e cuidados (D'Cruz \& Banerjee, 2020, Tyrrell \& Willians, 2020)

Vários artigos destacaram a inépcia do poder público que, durante anos, não fiscalizou ou sequer se preocupou em conhecer o que ocorria nos lares de idosos (no Brasil denominados instituições de longa permanência), onde a mortalidade entre as pessoas idosas foi particularmente acentuada. Muitos governos minimizaram o fato, destacando que a COVID-19, nestas situações, só era perigosa para os idosos. Ficou claro ainda, em alguns países, a resistência destas instituições em seguir diretrizes de saúde pública. A literatura destaca que estes comportamentos, se modificados, poderiam ter evitado várias mortes nestes locais (Academia Médica Francesa,2020; Fraser et al., 2020; Tarazona-Santalbina, Martinez-Velilla, \& Vidán, 2020; Wister \& Speechley, 2020).

Questões éticas significativas sobre quem deveria receber cuidados médicos intensivos ou suporte ventilatório ficaram muito evidentes nesta pandemia, principalmente em locais com poucos recursos de saúde ou onde estes últimos se encontravam sobrecarregados. Apesar de acaloradas discussões, decisões ad hoc foram tomadas pelos profissionais de saúde, inúmeras vezes pautadas pelo ageísmo (Ayalon et al., 2020)

Estas decisões foram permeadas por embates defendendo ou não o uso da idade como critério de maior probabilidade de morte ou de vida. A grande maioria dos geriatras e sociedades médicas se opuseram à decisão baseada apenas no critério de idade, sempre ressaltando a presença de ageísmo e de noções preconcebidas sobre o que seja a velhice quando se considera apenas os anos vividos, posto que, o ageísmo traz implícito que os idosos são menos resistentes e menos capazes de enfrentar desafios tais como o COVID-19 (Academia Médica Francesa, 2020; Colenda et al.,2020; Fraser et al., 2020).

Porém, em vários países, a idade foi formalmente adotada como critério de desempate para quando houvesse necessidade de decisão sobre o uso de ventiladores ou sobre a utilização de leitos de UTI, economizando assim recursos para 
aqueles que podem ter mais anos de vida, reproduzindo a visão de que o idoso é um fardo para a economia e para o sistema de saúde (Cesari \& Proietti, 2020; Fraser et al., 2020).

O ageísmo entre profissionais de saúde já vinha sendo discutido antes da pandemia, já que nada mais é que um reflexo da cultura. A literatura ressalta que estereótipos negativos sobre as pessoas idosas são perpetuados entre os profissionais de saúde pela sua própria formação, já que os alunos são expostos somente a idosos muito doentes, o que intensifica a associação de fragilidade com o envelhecer (Barrett, Michael, \& Padavic, 2020; Cesari \& Proietti, 2020; Fraser et al., 2020; Reynolds, 2020; Senger, 2020). Estes autores comentam que o critério idade surgiu como consequência lógica da formação médica, onde conceitos geriátricos são frequentemente ignorados.

Em artigo que discute se é errado ou não priorizar pacientes mais jovens com COVID-19, foram ouvidas opiniões contra e a favor de profissionais médicos do Reino Unido. Os que defendem a não utilização da idade como critério de desempate para o uso de ventiladores, reforçam que esta é uma visão ageísta, que associa o envelhecer ao morrer e à ausência de perspectivas de vida (Archard \& Caplan, 2020).

Negando o critério idade para alocação de tratamentos médicos, Archard (Archard \& Caplan, 2020) pergunta se este critério poderá ser então utilizado entre dois jovens: um de 18 e outro de 19 anos? Ou somente se aplica a idosos? Este autor destaca que se trata de uma visão de que a vida é apenas um bem, que pode e deve ser distribuído pela sociedade com base em critérios não muito claros e flutuantes de quem é merecedor ou não, atribuindo-se um juízo de valor arbitrário a quem teve ou não "uma boa vida". Esta é uma visão que passa um claro recado: a vida do idoso tem menos valor que as demais, estigmatizando-os como cidadãos de segunda classe.

Já Clapan (Archard \& Caplan, 2020), destaca que, devido à escassez de recursos, em países como a Itália, como exemplo, a idade foi adotada como critério, o que excluía pessoas com mais de 65 anos de cuidados intensivos. $\mathrm{O}$ autor destaca que o critério etário já vinha sendo utilizado por vários sistemas de saúde anteriormente à pandemia, citando como exemplo que, nestes locais, pessoas com mais de 80 anos não recebem transplantes. Para o autor, o critério etário se baseia no princípio de que cada pessoa deve ter oportunidade de viver uma vida e que pessoas mais velhas já tiveram esta oportunidade de perseguir seus objetivos e, portanto, devem ter menor prioridade.

Para aqueles que afirmam que não há outro critério prático para verificar, na presença de recursos escassos, quem priorizar, Cesari e Proietti (2020) ressaltam que há várias escalas geriátricas rapidamente aplicáveis em ambiente hospitalar que poderiam auxiliar a diferenciar condições clínicas entre os idosos, critério mais objetivo e inclusivo do que simplesmente idade. Porém, os autores afirmam que critérios ageístas foram os mais utilizados, apenas por sua suposta praticidade, demonstrando o pouco valor que a medicina moderna dá à vida humana. Gillett (2020) ressalta que não é possível quantificar previamente duração de vidas, nem as valorar a priori, pois elas não pertencem apenas à uma pessoa e sim influenciam todos um círculo de relações que serão impactadas por aquela morte. Assim, qualquer critério será injusto, porém, já que é necessário ter um, que seja o mais humano possível.

Não é apenas a idade o fator de risco para a mortalidade pelo COVID-19 e sim a presença de doenças crônicas e comorbidades são os fatores principalmente relacionados. Porém, como destaca o Escritório de Direitos Humanos das Nações Unidas (OMS, 2021), idosos com comprometimento cognitivo ou dependência física foram os que tiveram maior risco para ter sua autonomia e seus direitos violados. Destacando estes pontos, associações médicas europeias e norte-americanas se posicionaram veemente contra a aplicação do critério idade como determinante de risco ou acesso a cuidados médicos (Cox, 2020).

Por fim, autores (Hans-Joerg Ehni \& Hans-Werner Wahl, 2020; Monahan, MacDonald, Lytle, Apriceno, Levy, 2020; Wister \& Speechley, 2020; Pettretto \& Pili, 2020) listam proposições contra o ageísmo para o período pandêmico que deveriam ser sempre observadas. A primeira diz respeito a heterogeneidade do grupo etário idoso, entender esta diversidade é 
uma forma de contraposição aos estereótipos negativos. Já a segunda destaca que limitar acesso a recursos de tratamento apenas pela idade é antiético e inadequado. O terceiro ponto destaca a necessidade de fortalecer vínculos intergeracionais e o quarto ressalva a necessidade de não paternalizar a pessoa idosa. Estimular o uso de tecnologias de informação e comunicação pelos mais velhos é o quinto ponto destacado, sendo que, por último, assim como foi dado amplo destaque a virologia e farmacologia para o combate à COVID-19, a gerontologia deveria ter sido ouvida para uma melhor orientação política e entendimento da crise.

\section{Conclusão}

Mesmo com inúmeros esforços para combater o ageísmo e fomentar a solidariedade intergeracional, inúmeras manifestações surgiram e ainda se expressam incentivando estereótipos negativos contra as pessoas idosas. A pandemia tornou aparente e visível um fenômeno há muito presente na cultura. Se as restrições impostas pela COVID-19 buscaram proteger as pessoas idosas, acabaram por exacerbar estes estereótipos levando à abusos contra estas pessoas, aliados a outras formas de preconceito tal como o racismo ou capacitismo. Se por um lado é importante proteger as pessoas idosas da infecção, por outro é preciso respeitar este segmento etário enquanto sujeitos de direito, em sua autonomia e dignidade.

Ficou claro a naturalização da discriminação etária, reproduzida e exponencialmente propagada pelos governos, mídias e redes sociais. A homogeneização do grupo etário idoso e a visão de que apenas algumas vidas importam perpassa a pandemia de COVID-19. Descaso para com as instituições de longa permanência, generalizações excessivas, sugestões eticamente questionáveis foram pontos discutidos, em um discurso que contrapunha estereótipos à fatos científicos.

Apesar deste discurso, várias manifestações de solidariedade intergeracional ocorreram e ainda continuam ocorrendo, destacando a heterogeneidade do grupo etário idoso. Em um mundo onde as vulnerabilidades sociais, o acesso aos cuidados e a determinação social do adoecer se tornaram visíveis a todos, é obrigação enfrentar firmemente o ageísmo em todas as suas formas, oferecendo esperança, compaixão e empatia para com os grupos em desvantagem social, fortemente afetados pela COVID-19.

Destaca-se, por último, o pouco interesse que o tema despertou para a literatura acadêmica nacional, sendo muito mais amplamente analisado e discutido fora do Brasil, como o observado nesta revisão, o que limita a análise dos fatos que ocorreram no país. Esta limitação mostra-se, outrossim, como um espaço aberto para futuras pesquisas sobre o tema.

\section{Referências}

Academia Francesa de Medicina (2020). Ageism and intergenerational tensions in the COVID-19 period. Bull Acad Natl Med. https://doi.org/10.1016/j.banm.2020.05.048

Ângulo-Giraldo, M. A., Bonilla, G. R., Garcia, H. C., Colonia, L., \& Fernandez-Alvarado, S. (2020). Personas mayores en los medios digitales peruanos durante la pandemia por COVID-19. Kairós Gerontologia, 23, 2020.

Archard, D. \& Caplan, A. (2020). Is it wrong to prioritise younger patients with covid-19? BMJ ,369:m1509. 10.1136/bmj.m1509

Ayalon, L. (2020) There is nothing new under the sun: ageism and intergenerational tension in the age of the COVID-19 outbreak. International Psychogeriatrics: 1-4. doi:10.1017/S1041610220000575

Ayalon, L. et al. Aging in Times of the COVID-19 Pandemic: Avoiding Ageism and Fostering Intergenerational Solidarity. Journals of Gerontology: Psychological Sciences. doi:10.1093/geronb/gbaa051

Barrett, A. E., Michael, C. \& Padavic, I. (2020). Calculated Ageism: Generational Sacrifice as a Response to the COVID-19 Pandemic. Published by Oxford University Press on behalf of The Gerontological Society of America. Downloaded from https://academic.oup.com/psychsocgerontology/advancearticle/doi/10.1093/geronb/gbaa132/5897082 by guest on 28 September 2020

Bergman, Y. S., Cohen-Fridel, Sara, Shrira, A, Bodner, E., \& Palgi, Y. (2020). COVID-19 health worries and anxiety symptoms among older adults: the moderating role of ageism. Downloaded from https:/academic.oup.com/gerontologist/advance-article/doi/10.1093/geront/gnaa131/5904976

Bravo-Segal, S., \& Villar, F. (2020). La representación de los mayores en los medios durante la pandemia COVID-19: ¿hacia un refuerzo del edadismo? Rev Esp Geriatr Gerontol. 55(5):266-271 
Brooke, J. \& Jackson, D. (2020). Older people and COVID-19: Isolation, risk and ageism. Journal of Clinical Nursing. 29:2044-2046.

Cesari, M. \& Proietti, M. (2020) COVID-19 in Italy: Ageism and Decision Making in a Pandemic. JAMDA 21: 576e577. https://doi.org/10.1016/j.jamda.2020.03.025

Cohn-Schwartz, E. \& Ayalon, L. (2020). Societal views of older adults as vulnerable and a burden to society during the COVID-19 outbreak: Results from an Israeli nationally representative sample. Published by Oxford University Press on behalf of The Gerontological Society of America. Downloaded from https://academic.oup.com/psychsocgerontology/advance-article/doi/10.1093/geronb/gbaa150/5901136

Colenda, C. C. et al. (2020) COVID-19 Pandemic and Ageism: A Call for Humanitarian Care. Am J of Geriatric Psychiatry.28:805-807

Cox, C. (2020). Older Adults and Covid 19: Social Justice, Disparities, and Social Work Practice. Journal of Gerontological Social Work. https://doi.org/10.1080/01634372.2020.1808141

D'Cruz, M. \& Banerjee, D. (2020). An invisible human rights crisis: The marginalization of older adults during the COVID-19 pandemic - An advocacy review. Psychiatry Research. 292: 113369

Donizzetti, A. R. (2019). Ageism in an Aging Society: The Role of Knowledge, Anxiety about Aging, and Stereotypes in Young People and Adults. Int. J. Environ. Res. Public Health 2019, 16, 1329, doi:10.3390/ijerph16081329

Fraser, S. et al. (2020). Ageism and COVID-19: what does our society's response say about us? Age and Ageing, 49: 692-695. doi: 10.1093/ageing/afaa097

Guerios, A. L. (2019). Quarentenas do século XIV. Separar, para quê? Dividir para conquistar. Humanidades médicas

Gillett, G. (2020). COVID-19 Ethics—Looking Down the Muzzle. Journal of Bioethical Inquiry. https://doi.org/10.1007/s11673-020-10027-6

Hans-Joerg, E. \& Hans-Werner, W. (2020) Six Propositions against Ageism in the COVID-19 Pandemic, Journal of Aging \& Social Policy. 32:4-5, 515-525.

Hammerschimidt, K. S. A., Bonatelli, L. C. S. \& Carvalho, A. A. (2020). Caminho da esperança envolvendo os idosos: olhar da complexidade sob pandemia do COVID-19. Texto e Contexto de Enfermagem: pré-print.

Jimenez-Sotomayor, M. R., Gomez-Moreno, C. \& Soto-Perez-de-Celis, E. (2020). Coronavirus, Ageism, and Twitter: An Evaluation of Tweets about Older Adults and COVID-19. J Am Geriatr Soc 68:1661-1665.

Leão, L. R. B., Fereeira, V. H. S. \& Faustino, A. M. (2020). O idoso e a pandemia de COVID-19: uma análise de artigos publicados em jornais. Braz. J. of Develop. 6(7):451123-45142.

Lichtenstein, B. (2020). From "Coffin Dodger" to "Boomer Remover": Outbreaks of Ageism in Three Countries with Divergent Approaches to Coronavirus Control. Journals of Gerontology: Social Sciences. doi:10.1093/geronb/gbaa128

Manso, M. E. G., Mello, R. G. R., \& Lopes, R. G. C. (2018). Revista Observatório, 4 (2): 265-278

Moher, D, Liberati, A, Tetzlaff, J, Altman, Dg. The PRISMA Group (2009). Preferred Reporting Items for Systematic Reviews and Meta-Analyses: The PRISMA Statement. PLoS Med 6(7): e1000097.Doi:10.1371/journal.pmed1000097

Monahan, C., Macdonald, J., Lytle. A., Apriceno, M. B., \& Levy, S. R. COVID-19 and Ageism: How Positive and Negative Responses Impact Older Adults. American Psychological Association. 2 (999). http://dx.doi.org/10.1037/amp0000699

Moscovici, S. (2009). Representações sociais: investigações em Psicologia Social (6a ed.). Vozes.

Organização Mundial de Saúde [OMS]. (2019). A society is measured by how it cares for its elderly citizens. https:// www.who.int/news-room/featurestories/detail/a-society-ismeasured- by-how-it-cares-for-its-elderly-citizens.

Organização Mundial de Saúde [OMS]. (2021). Global campaign to combat ageism. Global report on ageism. Genebra, Suiça: OMS.

Petretto, D. R. \& Pili, R. (2020). Ageing and COVID-19: What Is the Role for Elderly People? Geriatrics, 5: 25, doi:10.3390/geriatrics5020025

Previtali, F., Allen, L. D. \& Varlamova, M. (2020) Not Only Virus Spread: The Diffusion of Ageism during the Outbreak of COVID-19. Journal of Aging \& Social Policy, 32:4-5, 506-514

Reynolds, L. M. A. (2020). The COVID-19 Pandemic Exposes Limited Understanding of Ageism. Journal of Aging \& Social Policy. 32 (4-5): 499-505

Senger, E. (2020) Ageism in medicine a pressing problem. CMAJ, 14,191:E55-6. doi: 10.1503/cmaj.109-5698

Sepúlveda-Loyola, W. et al. (2020). Impact of Social Isolation Due to COVID-19 on Health in Older People: Mental and Physical Effects and Recommendations. J Nutr Health Aging. 24(9):938-947. doi: 10.1007/s12603-020-1469-2.

Silva, S. P. et al. (2020). Idoso, COVID-19 e mídia jornalística. Revista Kairós-Gerontologia, 23: 287-307.

Tarazona-Santabalbina, F. J., Martínez-Velilla, N., Vidán, M. T., \& García-Navarro, J. A. (2020). COVID-19, adulto mayor y edadismo: errores que nunca han de volver a ocurrir. Rev Esp Geriatr Gerontol. 55(4):191-192

Teixeira, S. M. O., Souza, L. E. C., \& Maia, L. M. (2018). Ageísmo institucionalizado: uma revisão teórica. Revista Kairós-Gerontologia. 21(3):129-149

Tyrrell, C. J. \& Williams, K. N. (2020). The Paradox of Social Distancing: Implications for Older Adults in the Context of COVID-19. American Psychological Association 2020. 12 (1): 214-S216. http://dx.doi.org/10.1037/tra0000845 
Research, Society and Development, v. 10, n. 11, e274101119233, 2021

(CC BY 4.0) | ISSN 2525-3409 | DOI: http://dx.doi.org/10.33448/rsd-v10i11.19233

Vervaecke, D. \& Meisner, B. (2020). Caremongering and Assumptions of Need: The Spread of Compassionate Ageism During COVID-19. International Psychogeriatrics: 1-5. doi:10.1017/S1041610220001258

Wister, A. \& Speechley, M. (2020). COVID-19: Pandemic Risk, Resilience and Possibilities for Aging Research. Canadian Journal on Aging / La revue canadienne du vieillissement. 39 (3):344-347. doi:10.1017/S0714980820000215

Xiang, X, Lu, X, Halavanau, A., Xue, J., Sun, Y., Lam Lai, P. H., \& Wu, Z. (2020). Modern Senicide in the Face of a Pandemic: An Examination of Public Discourse and Sentiment About Older Adults and COVID-19 Using Machine Learning. J Gerontol B Psychol Sci Soc Sci, 2020: 1-11. doi:10.1093/geronb/gbaa128 